\title{
Recent Treatment of Data in Chemical Analysis
}

\author{
Takashiro Akitsu* and Atsuo Yamazaki \\ Department of Chemistry, Tokyo University of Science, Tokyo, Japan
}

Corresponding author: Takashiro Akitsu, Professor of Chemistry, Department of Chemistry, Faculty of Science, Tokyo University of Science, 1-3 Kagurazaka, Shinjuku-ku, Tokyo 162-8601, Japan, Tel: +81-3-5228-8271; Fax: +81-3-5261-4631; E-mail: akitsu@rs.kagu.tus.ac.jp

Copyright: (c) 2017 Akitsu T, et al. This is an open-access article distributed under the terms of the Creative Commons Attribution License, which permits unrestricted use, distribution, and reproduction in any medium, provided the original author and source are credited.

Citation: Akitsu T, Yamazaki A. Recent treatment of data in chemical analysis. Trends Green Chem. 2017, 3:2.

\section{Editorial}

Not only in green chemistry but also in other fields of chemistry, there is a problem of analytical values spreading an error and noise in the case of arguing the data of substances or compounds thoroughly. Therefore, we have considered to establish a method of good assessed values based on a science of statistics for experimental data [1] taken from students' experiments of chemistry [2] including various types of analysis.

According to general discussion by Bayes's theorem, attracting attention recently, when a theoretical framework $T$ is give, a product of probability $p(T \mid E) \propto p(T) p(E \mid T)$ can be assumed where $p(E \mid T)$ is probability after experiments, $p(T)$ is probability before experiments. Moreover, average, median, mode as the best estimated values will be within standard deviation (std) deviated from average in many cases. Thus, all the three values may be the best estimated values. Based on this theoretical background, we attempted to compare data of certain years by using two data groups (1) required amount of sodium hydroxide as before experiments (2) resulting concentration of oxalic acid as actual experimental results during neutralizing titrates (theoretical framework).

For example, neutralizing titrates of oxalic acid (weak acid) by sodium hydroxide (strong base) exhibited average required amount of sodium hydroxide was $11.3 \mathrm{~mL}$ (std 1.91) and $\mathrm{pH} 6.91$ (std 0.42 ) in a certain year. Different results were obtained in another year (Table 1) or using different indicators, though theoretical framework (experiments of neutralizing titrates) was identical.

Table 1 Oxalic acid experimental results for 4 years.

\begin{tabular}{|l|l|l|l|l|}
\hline Year & $\mathbf{2 0 1 0}$ & $\mathbf{2 0 0 9}$ & $\mathbf{2 0 0 8}$ & $\mathbf{2 0 0 7}$ \\
\hline no. of data & 32 & 32 & 36 & 32 \\
\hline average & 0.049721 & 0.044708 & 0.045178 & 0.050472 \\
\hline std & 0.004418 & 0.010489 & 0.010472 & 0.000583 \\
\hline median & 0.0507 & 0.050131 & 0.050772 & 0.050477 \\
\hline frequency & 0.06 & 0.06 & 0.06 & 0.06 \\
\hline
\end{tabular}

\section{References}

1. Atsuo Yamazaki, Yosuke Mori, Maiko Ito, Takashiro Akitsu (2015) Poster presentation in 5th Chemistry Festa by the chemical society of Japan.

2. Department of Chemistry, Faculty of Science, Tokyo University of Science, Textbook of inorganic and analytical chemistry experiments 2015 edition, 2015. 